\title{
On the Low-Complexity Soft-Decoding of Binary Quadratic Residue Codes
}

\author{
Tsung-Ching Lin, Wen-Ku Su*, Pei-Yu Shih, and Trieu-Kien Truong, IEEE Fellow \\ Department of Information Engineering, I-Shou University, Kaohsiung County 84001, Taiwan \\ *Corresponding Author: d9403003@isu.edu.tw
}

\begin{abstract}
This paper is to shown the performance of a modified algorithm for decoding the $(47,24,11)$ binary quadratic residue code up to six errors. The technique in this paper combines the algebraic decoding algorithm with the detection scheme for the $(47,24,11)$ quadratic residue code offered by Truong et al. to correct up to five errors. Then again, the reliability-search algorithm is utilized to correct one bit of the six bits error. The computer simulation of the scheme shows that at least $90 \%$ of 6 bits error occurred are corrected if the $\mathrm{E}_{\mathrm{b}} / \mathrm{N}_{0}$ ratios are greater than $4 \mathrm{~dB}$. As a result, the performance of this modified algorithm is very close to the bound of decoding up to six-bit error.
\end{abstract}

Keywords: soft-decision decoding; syndrome matrix; Berlekamp-Massey algorithm; quadratic residue code; errorlocator polynomial; error detection.

\section{Introduction}

The quadratic residue $(\mathrm{QR})$ codes are introduced by Prange (1), in 1958, which are one class of cyclic codes. Most of the known QR codes are as good as the best code with the code length $n$ and the dimension $k$, see Fig. 5.2 of (2). Among them, the $(47,24,11)$ QR code is one of the best codes, and has the five error-correcting capacity. The $(48,24,12)$ extended QR code is formed by adding a parity-check bit $p$ to a codeword of the $(47,24,11)$ QR code. The $(48,24,12)$ extended QR code thus has the capability to correct five errors and detect six errors. Moreover, this extended QR code is also the extremal self-dual doubly-even code (3).

In this paper, the algebraic decoding algorithm for the $(47,24,11)$ QR code proposed in (4) is developed to decode up to five errors and can be utilized to decode the $(48,24,12)$ QR code for correcting five errors and detecting six errors. Recently, the reliability-search algorithm in (5) was developed to facilitate further decoding of the $(23,12,7)$ Golay code. In that algorithm, using real channel data, the method developed by Reed (6) can be used to estimate the individual bit-error probabilities in a received word. It is convenient to let the symbols $\boldsymbol{D}_{v}$ and $\boldsymbol{D}$ denote the algebraic decoding algorithm of the $(47,24,11)$ QR code (4) for $v$ errors and up to five errors, respectively. In this paper, the performance of decoding the $(47,24,11)$ extended QR code by using a modified decoding algorithm which is developed via the $\boldsymbol{D}$ algorithm and the reliability-search algorithm (5) is shown.

\section{Terminology and background of $(47,24,11)$ QR code}

Consider the $(47,24,11)$ binary $Q R$ code with code length 47 defined over $G F\left(2^{23}\right)$ whenever $47 \mid 2^{23}-1$. Let $Q_{47}$ denote the set of quadratic residues modulo a prime integer 47 , i.e., $Q_{47}=\left\{i^{2}(\bmod 47) \mid 0 \leq i \leq(47-1) / 2\right\}=\{1,2,3,4,6,7,8$, $9,12,14,16,17,18,21,24,25,27,28,32,34,36,37,42\}$. Let $\beta$ be a primitive 47 -th root of unity such that $x^{47}-1=$ where $Z_{47}$ is the integer ring modulo 47 , i.e., $Z_{47}=\{0,1, \ldots$, $46\}$. The $(47,24,11)$ binary QR code is defined be a cyclic code with the following generator polynomial,

$$
g(x)=\prod_{i \in Q_{47}}\left(x-\beta^{i}\right)
$$

Let codeword $c(x)=m(x) g(x)$ be transmitted through a noisy channel to obtain a received word of form, $r(x)=c(x)+e(x)$, where $e(x)$ is the error polynomial. The syndromes $S_{i}$ of the received word $r(x)$ are defined as $S_{i}=r\left(\beta^{i}\right)$ and are expressible in terms of the following symmetric polynomials:

$$
S_{i}=Z_{1}^{i}+Z_{2}^{i}+\cdots+Z_{v}^{i}, \text { for } 1 \leq i<47,
$$

where $Z_{j}$ for $0 \leq j<v$ are the locations of the $v$ errors, i.e., $Z_{j}$ $=\beta^{r_{j}}$ with $r_{j}$ being the location of the error to be corrected and $v \leq t$. If the syndromes can be computed from

$$
S_{i}=r\left(\beta^{i}\right) \text { where } i \in Q_{47},
$$

these are called the known syndromes. Otherwise, the syndromes are called the unknown syndromes.

Next, define the error-locator polynomial of the error pattern $e(x)$ to be 


$$
L(z)=\prod_{i=1}^{v}\left(z-Z_{i}\right)=z^{v}+\sum_{j=1}^{v} \sigma_{j} z^{v-j}
$$

where

$$
\sigma_{i}=\sum_{0 \leq j_{1}<j_{2} \cdots<j_{v} \leq v} Z_{j_{1}} Z_{j_{2}} \cdots Z_{j_{v}} \quad(1 \leq i \leq v) .
$$

Then, the relations of $S_{i}$ and $\sigma_{j}$ are the Newton's identities shown in (7) given in the following:

$$
\begin{array}{ll}
S_{i}+\sum_{j=1}^{i-1} \sigma_{j} S_{i-j}+\sigma_{i}=0, & (1 \leq i \leq v, i \text { is odd }) \\
S_{i}+\sum_{j=1}^{i-1} \sigma_{j} S_{i-j}=0, & (1 \leq i \leq v, i \text { is even }) \\
S_{i}+\sum_{j=1}^{v} \sigma_{j} S_{i-j}=0, & (i \geq v)
\end{array}
$$

The error-locator polynomial could be obtained by directly solving the Newton's identities for the $\sigma_{j}, 1 \leq j \leq v$. If there are sufficient numbers of consecutive known syndromes for a given number of errors, we can gain the error-locator polynomial via the Berlekamp-Massey algorithm. In the following, the hard decoding algorithm of the $(47,24,11) \mathrm{QR}$ code and the detection the six bits error method for its extended code are introduced.

\section{Algebraic decoding algorithm for the $(47,24$, 11) $Q R$ code and detection six bits error for its extended code}

Depending on the number of $v=0,1,2,3$ errors, the coefficients of the error-locator polynomial are defined in Case 0, Case 1, Case 2, and Case 3, respectively. For $v=4$ and 5 , we first define the unknown syndromes $S_{5}$ by using the syndrome matrices. Then there are sufficient numbers of consecutive known syndromes, $S_{0} S_{1}, \ldots, S_{7}, S_{8}$, and we can gain the error-locator polynomial via the Berlekamp-Massey algorithm.

Case 0: If $S_{1}=0$, then there is no error occurred.

Case 1: If $S_{1}^{47}=1$, there is one bit error occurred and the error-locator polynomial is $L(z)=z+S_{1}$.

Case 2: If there are two bits error occurred, the errorlocator polynomial is $L(z)=z^{2}+S_{1} z+S_{3} / S_{1}+S_{2}$.

Case 3: If $S_{7}+S_{6} S_{1}=0$, there are more than three bits error occurred and go to Case 4. Otherwise, the coefficients of $L(z), \sigma_{1}, \sigma_{2}$, and $\sigma_{3}$, are obtained:

$\sigma_{1}=S_{1}$

$\sigma_{2}=\frac{S_{9}+S_{1}^{9}+S_{6}\left(S_{3}+S_{1}^{3}\right)}{S_{7}+S_{6} S_{1}}$,

$$
\sigma_{3}=S_{3}+S_{1}^{3}+\frac{\left(S_{9}+S_{1}^{9}\right) S_{1}+S_{6}\left(S_{3}+S_{1}^{3}\right) S_{1}}{S_{7}+S_{6} S_{1}} .
$$

In Cases 4 and 5, the technique in (6) for finding the value of unknown syndrome $S_{5}$ is used and one shows the matrices $S(I, J)$. By the $\operatorname{det}(S(I, J))=0$, the $S_{5}$ 's value is determined. Then, sufficient consecutive known syndromes are used to solve the Newton's identities. Moreover, the coefficients $\sigma_{1}, \sigma_{2}, \sigma_{3}, \sigma_{4}$, and $\sigma_{5}$ are obtained via BerlekampMassey algorithm.

Case 4: For the four bits error, $S_{0}=0$. The matrix $S(I, J)$ is given:

$$
S(I, J)=\left[\begin{array}{ccccc}
S_{1} & S_{3} & S_{4} & S_{14} & S_{21} \\
S_{4} & S_{6} & S_{7} & S_{17} & S_{24} \\
S_{5} & S_{7} & S_{8} & S_{18} & S_{25} \\
\hline S_{14} & S_{16} & S_{17} & S_{27} & S_{34} \\
S_{34} & S_{36} & S_{37} & S_{0} & S_{7}
\end{array}\right] .
$$

The entries of $S(I, J)$ are known except $S_{5}$. Next, $\operatorname{det}(S(I, J))=0$. Thus, $S_{5}$ can be solved as follows:

$$
S_{5}=\frac{\operatorname{det}\left(\Delta_{0}\right)}{\operatorname{det}(\Delta)}
$$

$$
\text { where } \begin{aligned}
\Delta & =\left[\begin{array}{ccccc}
S_{1} & S_{3} & S_{4} & S_{14} & S_{21} \\
S_{4} & S_{6} & S_{7} & S_{17} & S_{24} \\
\underline{0} & S_{7} & S_{8} & S_{18} & S_{25} \\
S_{14} & S_{16} & S_{17} & S_{27} & S_{34} \\
S_{34} & S_{36} & S_{37} & S_{0} & S_{7}
\end{array}\right] \text { and } \\
\Delta_{0} & =\left[\begin{array}{llll}
S_{3} & S_{4} & S_{14} & S_{21} \\
S_{6} & S_{7} & S_{17} & S_{24} \\
S_{16} & S_{17} & S_{27} & S_{34} \\
S_{36} & S_{37} & S_{0} & S_{7}
\end{array}\right]
\end{aligned}
$$

If $\operatorname{det}(\Delta)=0$, there must be more than four bits error.

Case 5: For five bits error, $S_{0}=1$ and $S_{5}$ is determined by using following matrices.

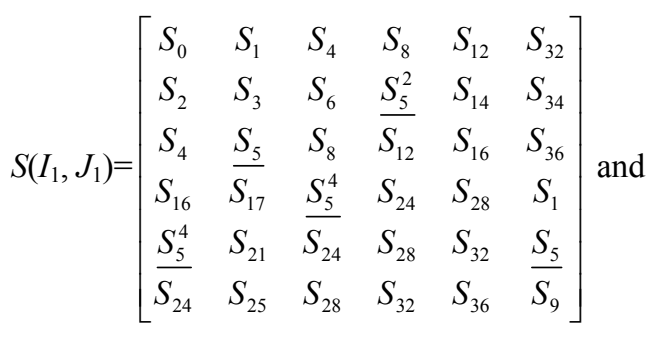




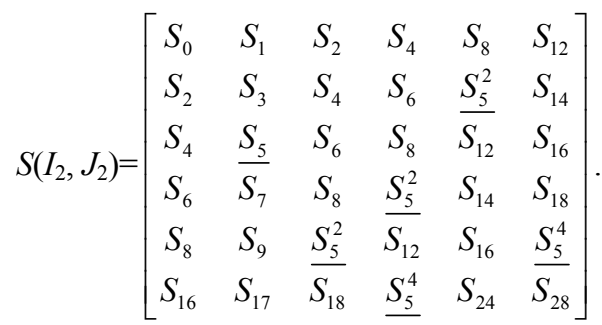

Obviously, $S_{5}$ is the root of the greatest common divisor of $\operatorname{det}\left(S\left(I_{1}, J_{1}\right)\right)$ and $\operatorname{det}\left(S\left(I_{2}, J_{2}\right)\right)$.

Furthermore, the roots of $L(z)$ belong to $\left\{\beta^{0}, \beta^{1}, \ldots, \beta^{46}\right\}$. Moreover, the Chien's search is used to find the roots of the error-locator polynomial. The error locations are the roots of $L(z)$, and the error pattern is determined.

Consider the binary $(48,24,12)$ extended QR code $C^{\prime}$. $\boldsymbol{r}$ is the received word without its overall parity bit $\boldsymbol{p}$. In the $\boldsymbol{D}$ algorithm, first the syndromes of $\boldsymbol{r}$ are calculated and the number of the error occurred is determined by the above mentioned cases. Then either the roots of $L(z)$ are the error locations found by the Chien's search and the error is thus obtained or not. If $\boldsymbol{D}$ can be used to compute the error $\boldsymbol{E}_{\boldsymbol{D}}$ from the known $\boldsymbol{r}$, the decoding scheme of the code augmented by a parity bit, called extended $\boldsymbol{P}$, is given as follows:

1) If the weight of $\boldsymbol{E}_{\boldsymbol{D}}$, notation $w t\left(\boldsymbol{E}_{\boldsymbol{D}}\right)$, is less than 5, then $\boldsymbol{r}$ can be decoded as $\boldsymbol{r}+\boldsymbol{E}_{\boldsymbol{D}}$ and compute the parity $\boldsymbol{p}$.

2) If the $w t\left(\boldsymbol{E}_{\boldsymbol{D}}\right)=5$, then compute the parity $\boldsymbol{p}$ of $\boldsymbol{r}+\boldsymbol{E}_{\boldsymbol{D}}$ and compared with the received parity $\boldsymbol{p}$. If $\boldsymbol{p} \neq \boldsymbol{p}$, then the decoder detects a six bits error.

If no error is obtained from $\boldsymbol{r}$ by the $\boldsymbol{D}$ algorithm or a six bits error is detected by extended $\boldsymbol{P}$, one uses a method mentioned below to correct the six bits error.

\section{Reliability-search algorithm to correct the six bits error}

In (5), Dubney et al. proposed an improved version of the previous shift-search algorithm by using the bit-error probability estimate that is developed by Reed et al. (6). In the algorithm, they add a selected one bit error to the received word and then decode that obtained word, where the selection of the added error is based on the probability calculated from the received word. If it can be decoded successfully, then the actually "codeword" is just the output word. If it cannot be decoded, try another one bit error having the probability only lower than the previous selection. If all the possible selections do not offer decodable word, the actually error occurred in the received word has weight larger than the error-correcting capacity and is not decodable.

For each $j$, let $p^{j}$ denote the $j$-th bit error probability of the received word. Rearrange the sequence $p^{j}$ 's in decreasing order, namely, $p^{j_{1}} \geq p^{j_{2}} \geq \cdots \geq p^{j_{48}}$. Suppose that exactly a six bits error occurs in the received word. The method that uses the reliability-search algorithm and the algebraic decoding algorithm to correct six errors for the $(48,24,12)$
QR code consists of five steps:

Step1) Calculate the bit-error probabilities $p^{1}, p^{2}, \cdots, p^{48}$ of the received word and arrange them in decreasing order, i.e. $p^{j_{1}} \geq p^{j_{2}} \geq \cdots \geq p^{j_{48}}$.

Step 2) Let $k=1$.

Step 3) If the bit with the $k$-th highest probability of error belongs to $\boldsymbol{E}_{\boldsymbol{D}}$ or is the parity check bit, decode $\boldsymbol{r} \mid \boldsymbol{p}$ as $\left(\boldsymbol{r}+\boldsymbol{E}_{\boldsymbol{D}}\right) \mid(\boldsymbol{p}+1)$ and stop.

Step 4) Obtain $r$ ' by inverting the $j_{k}$-th bit of the received word. If a five bits error $\boldsymbol{E}_{\boldsymbol{D}_{5}}$ is obtained by the $\boldsymbol{D}_{5}$ algorithm, decode $\boldsymbol{r} \mid \boldsymbol{p}$ as $\left(\boldsymbol{r}+\boldsymbol{E}_{\boldsymbol{D}_{5}}\right) \mid \boldsymbol{p}$ and stop.

Step 5) If $k<48, k=k+1$ and return to Step 3. Otherwise, stop.

During the experiments, we observe that, in most cases, the inverted bit that cancels the sixth error can be correctly indicated within three attempts by using the reliability-search algorithm. The simulation results in additive white Gaussian noise (AWGN) illustrated in Table I show that at least $90 \%$ of a six bits error are corrected within three attempts if the $E_{b} / N_{0}$ ratios are greater than $4 \mathrm{~dB}$. Moreover, as shown in Fig. 1, One observes that the Frame Error Rate(FER) performance of the extended $(48,24,12) \mathrm{QR}$ code within three attempts is very close to the bound of the decoding up to six bits error.

\section{Conclusion}

The decoding algorithm can be used to correct very large percent of six bits error and all five bits errors or fewer random errors. Note also that as a bit-energy to noise-spectraldensity ratio(SNR) increases, the percentage of patterns of six bits error, which are decoded successfully, is improved. As a result, the performance of this algorithm is very close to the bound of decoding up to six-bit error. Furthermore, the method introduced in this paper can be generalized to decode for other QR codes more than their error-correcting capacity.

\section{Acknowledgment}

This work was supported by National Science Council, R.O.C., under Grant NSC101-2221-E-214-040.

\section{References}

(1) E. Prange : "Some cyclic error-correcting codes with simple decoding algorithms," Air Force Cambridge Research Center-TN-58-156, 1958.

(2) R. E. Blahut: Theory and Practice of Error Control Code. Reading, MA: Addison-Wesley, 1983.

(3) S.K. Houghten, C.W.H. Lam, L.H. Thiel, and J.A. Parker : "The extended quadratic residue code is the only $(48,24,12)$ self-dual doubly-even code", IEEE Trans. Inform. Theory, vol. 49, pp. 53 - 59, Jan. 2003.

(4) R. He, I. S. Reed, T. K. Truong, and X. Chen : "Decoding of the $(47,24,11)$ quadratic residue code," IEEE Trans. Inform. Theory, vol. 47, pp. 1181-1186, Mar. 2001. 
(5) G. Dubney and I. S. Reed : "Decoding the $(23,12,7)$ Golay code using bit-error probability estimates," Proceedings of IEEE Global Telecommunications Conference, November 2005.

(6) I. S. Reed : 'Statistical error control of a realizable binary symmetric channel'. Group Report 47.35, Massachusetts
Institute of Technology, Lincoln Laboratory, Massachusetts, Nov. 1959.

(7) F. J. Macwilliams and N. J. A. Sloan : The Theory of Error-Correcting Codes. Amsterdam, The Netherlands: North-Holland, 1977.

Table 1. The Percentage of Successful Decoding for Four Bits Error Occurred at SNR of 0 to $7 \mathrm{~dB}$ in AWGN

\begin{tabular}{c|cccccccc}
\hline \hline SNR(dB) & $\mathbf{0}$ & $\mathbf{1}$ & $\mathbf{2}$ & $\mathbf{3}$ & $\mathbf{4}$ & $\mathbf{5}$ & $\mathbf{6}$ & $\mathbf{7}$ \\
\hline $\begin{array}{c}\mathbf{\%} \\
\text { (1 Attempt) }\end{array}$ & 42.20 & 47.73 & 54.07 & 60.99 & 86.22 & 77.31 & 84.67 & 90.86 \\
\hline $\begin{array}{c}\mathbf{\%} \\
\text { (2 Attempt) }\end{array}$ & 61.91 & 67.67 & 74.04 & 80.08 & 90.62 & 91.29 & 95.18 & 97.67 \\
\hline $\begin{array}{c}\text { \% } \\
\text { (3 Attempt) }\end{array}$ & 71.12 & 76.24 & 81.56 & 86.17 & 91.76 & 94.03 & 96.50 & 98.10 \\
\hline
\end{tabular}

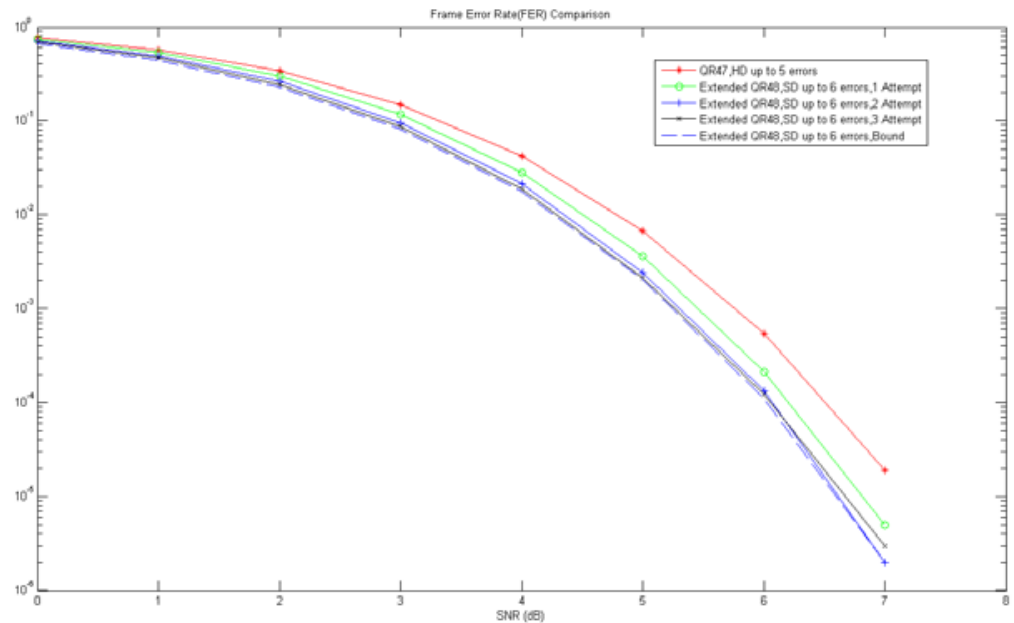

Fig. 1. Frame Error Rate Comparison of the (HD vs SD) Decoding of Quadratic Residue Code(QR). 